\title{
Effect of a Supplement Containing Olive Leaf Extract against Ultraviolet Light-Induced Tanning: A Double-Blind Placebo-Controlled Study
}

\author{
Izuru Kaneko, Tomohiro Chiba, Kohsuke Hayamizu, Tomoko Tsuji
}

FANCL Research Institute, FANCL Corporation

\begin{abstract}
Numerous studies have been conducted to research substances useful for preventing or reducing pigmentation in the skin. Pigment spot is a serious cosmetic concern, particularly for the Asian elderly. Oral ingestion of antioxidant supplements is known to prevent skin pigmentation. Glutathione is an intracellular antioxidant that plays an important role in cell protection against oxidative stress and shows antimelanogenic activity. In our previous study, it was shown that olive leaf extract stimulated intracellular glutathione synthesis in human fibroblasts, melanocytes and keratinocytes. Here, a double-blind placebo-controlled study was performed to investigate whether the ingestion of a supplement containing olive leaf extract, Lcystine and ascorbic acid affects tanning of skin induced by ultraviolet (UV) irradiation. We determined the minimal erythema dose (MED) in 24 healthy volunteers before and after supplementation. Small sites on the subjects' arms were exposed to UV light for three successive days, and subjects were then administered tablets containing $47.7 \mathrm{mg}$ olive leaf extract, $40.0 \mathrm{mg} \mathrm{L}-$ cystine and $50.0 \mathrm{mg}$ ascorbic acid every day for 8 weeks. The present study showed that the subjects' MED increased significantly after an 8-week course of supplementation. The increment of skin lightness at the sites of UV irradiation from Day 10 to Day 17 was significantly higher in the test sample group than in subjects who received a placebo. These results suggest that the supplement containing olive leaf extract, L-cystine and ascorbic acid reduces UV-induced erythema and tanning by its antioxidative activity.
\end{abstract}

$\boldsymbol{K} \boldsymbol{E} \boldsymbol{Y} \boldsymbol{W O R D S}$ : olive leaf extract, hydroxytyrosol, glutathione, tanning, UV irradiation

\section{Introduction}

Pigment spot in the skin is one of the most common cosmetic problems, especially for the aged. It is known that pigmentation is induced and exacerbated by ultraviolet (UV) light, and that UV light produces reactive oxygen species (ROS), which then stimulate melanocytes and promote melanogenesis. ${ }^{1-3)}$ Therefore, it is important to inhibit the production of ROS in order to prevent skin pigmentation.

Glutathione $(\mathrm{GSH})$ is an intracellular antioxidant that plays an important role in protecting cells against oxidative stress by directly scavenging ROS and participating in the GSH peroxidasecatalysed reaction, thereby eliminating hydrogen peroxide and other organic peroxides. ${ }^{4)}$ Moreover, GSH reinforces the reductive efficacy of ascorbic acid by reconverting dehydroascorbic acid into reduced ascorbic acid. It may also switch the melanogenesis type from eumelanin to pheomelanin. ${ }^{5,6)}$ It has been reported that GSH in the skin is depleted by UV light. ${ }^{7,8)}$ Intracellular GSH also decreases with aging, ${ }^{8,9)}$ and thus its protective intracellular functions are lost. This is one reason why abnormal skin pigmentation becomes more common with age. An increase in intracellular GSH levels could enhance the photo-protective effect of cells through several mechanisms, although it has not yet been confirmed whether or not oral ingestion of GSH increases intracellular GSH levels. In our study, we tried to find substances that increase intracellular GSH, and found that olive leaf extract does so in human fibroblasts, melanocytes and keratinocytes. Olive leaf extract is utilized in complementary and alternative medicine in Europe, ${ }^{10)}$ where it is commonly used against various infectious diseases and has been reported to have antioxidant properties.

We speculated combining the olive leaf extract and cystine is more effective than applying olive leaf extract alone to increase intracellular GSH, because GSH is synthesized from cystein, which is converted from cystine. So we performed a double-blind, placebo-controlled clinical study to investigate whether the ingestion of a supplement containing olive leaf extract, L-cystine and ascorbic acid prevents tanning in humans. 


\section{Methods}

\section{Subjects}

Healthy volunteers were recruited for an 8-week, double blind placebo-controlled clinical study designed to investigate the tanning prevention effect of a supplement containing olive leaf extract, L-cystine and ascorbic acid. Subjects who had a history of photosensitivity were excluded from the study. All subjects gave written informed consent before participating, and the study was approved by the Institutional Review Board of FANCL Co. (Yokohama, Japan) and carried out in accordance with the Declaration of Helsinki of 1964 (revised in 2000).

\section{Samples}

To produce the olive leaf extract, dried olive leaf was first extracted by ethanol, then hydrolyzed by acid, and finally concentrated and dried. A test sample tablet contained $47.7 \mathrm{mg}$ olive leaf extract, $40.0 \mathrm{mg}$ L-cystine and $50.0 \mathrm{mg}$ ascorbic acid. The placebo which was comprised of vehicles was an indistinguishable tablet from the test sample.

\section{Study methods}

A Multiple Solar Ultraviolet Simulator Model 601 (Solar Light Co., Philadelphia, PA, USA) consisting of a $150 \mathrm{~W}$ xenon arc lamp with a spectrum similar to sunlight was used to irradiate a site $9 \mathrm{~mm}$ in diameter. The intensity of the light at the skin surface was measured with an Erythema UV \& UVA Intensity Meter Model 3D-600 V2.0 (Solar Light Co.). For each subject, the minimal erythema dose (MED), defined as the smallest dose inducing erythema with a sharp border at 24 hours after irradiation, was determined both before the start of the study and after an 8week administration of the test sample or placebo. Four sites on the left upper inner arm were irradiated for 3 min with 25, 30, 40 and $50 \mathrm{~mJ} / \mathrm{cm}^{2}$ of UV light, and $60 \mathrm{~mJ} / \mathrm{cm}^{2}$ was added if no doses induced erythema. Doses of $0,0.75$ and 1 MED were then irradiated on three sites on the right upper inner arm of each subject three times daily for the first three days, giving a total daily exposure dose of $0,2.25$ and 3 MED, respectively.

The study design was carried out in a double-blind, placebocontrolled manner. Subjects were assigned to the test sample group or the placebo group with no differences in age, sex or initial MED. Subjects took 2 tablets of either the test sample or placebo three times daily after each meal for 8 weeks beginning on the first day of UV irradiation, but were not told which tablet they had taken. The skin lightness ( $\mathrm{L}^{*}$ value) of the UV irradiation sites on the right upper inner arm was measured by Minolta Chroma Meter CM-508d (Minolta Co. Ltd., Tokyo, Japan) at 0, 3, 10, 17, 31, 45 and 59 days after the first irradiation. The measurer was also blinded to the subject's group assignment.

\section{Statistical analysis}

Results are presented as mean $\pm \mathrm{SE}$. The differences in MED and $\mathrm{L}^{*}$ values between the test sample and placebo groups were analyzed using Student's t-test. The changes of MED from the initial values were determined by paired t-test. $\mathrm{P}<0.05$ was considered to be statistically significant. Statistical analysis was performed with StatView Ver. 5.0 for Windows (SAS Institute Inc., Cary, NC, USA).

\section{Results}

This study enrolled 24 healthy volunteers ( 20 men, 4 women), with no differences in age, sex or initial MED between the test sample group and the placebo group (Table 1). All subjects completed the course, but two subjects (1 man and 1 woman) from the placebo group missed an appointment to determine their MED after the treatment for personal reasons. No subjects were eliminated from the study protocol for any adverse event related to treatment.

\section{Table 1 Characteristics of subjects by study group}

\begin{tabular}{|c|c|c|}
\hline & Test sample group & Placebo group \\
\hline Subject; Total (Men / Women) ${ }^{a}$ & $13(11 / 2)$ & $11(9 / 2)$ \\
\hline Age (years) ${ }^{b}$ & $31.2 \pm 1.6$ & $32.1 \pm 1.6$ \\
\hline Initial MED $\left(\mathrm{mJ} / \mathrm{cm}^{2}\right)^{b}$ & $40.4 \pm 3.0$ & $37.8 \pm 3.1$ \\
\hline
\end{tabular}

$\mathrm{a}:$ Number, $\mathrm{b}$ :Mean and SE

There was no significant difference in the MEDs of the placebo group and the test sample group after the treatment. However, in the test sample group, the MED increased significantly after ingestion of the tablets as compared with before ingestion (from $40.4 \pm 3.0$ to $42.0 \pm 3.1 \mathrm{~mJ} / \mathrm{cm}^{2}$ ) while no significant difference was observed between MEDs before and after treatment in the placebo group (from $37.8 \pm 3.1$ to $38.9 \pm 3.4 \mathrm{~mJ} / \mathrm{cm}^{2}$ ) (Fig. 1).
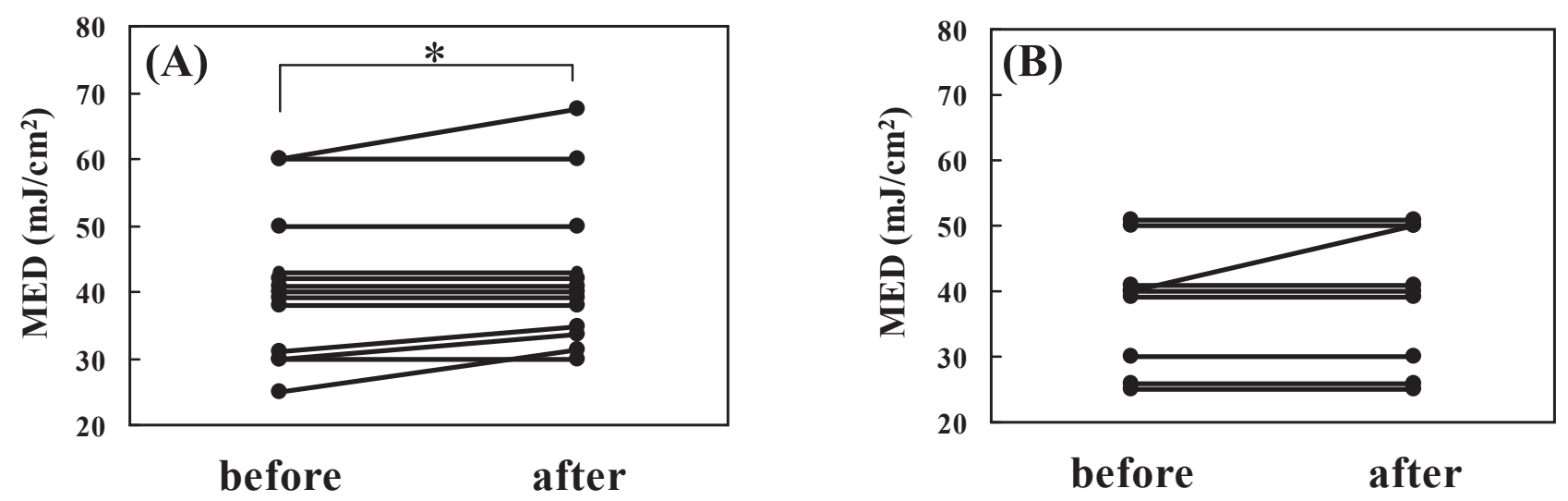

Fig. 1. Changes in the minimal erythema dose (MED) before and after 8 weeks of administration of test sample or placebo tablets. (A): test sample group (B): placebo group. Paired t-test. *: $\mathrm{p}=0.05$. 
The lightness ( $\mathrm{L}^{*}$ value) of the sites of UV irradiation measured the lowest 10 days after irradiation. The $\mathrm{L}^{*}$ value of the test sample group was slightly higher than that of the placebo group but was not statistically significant at that point (Fig. 2). After Day 10 , the $\mathrm{L}^{*}$ value increased gradually in both groups, with the increment of $\mathrm{L}^{*}$ value during the period between Day 10 and Day 17 measuring significantly higher in the test sample group irradiated with 3 MED than in the corresponding placebo group (Fig. 3). No significant difference was observed between the test sample group and the placebo group in the intact sites or at those irradiated with 2.25 MED.

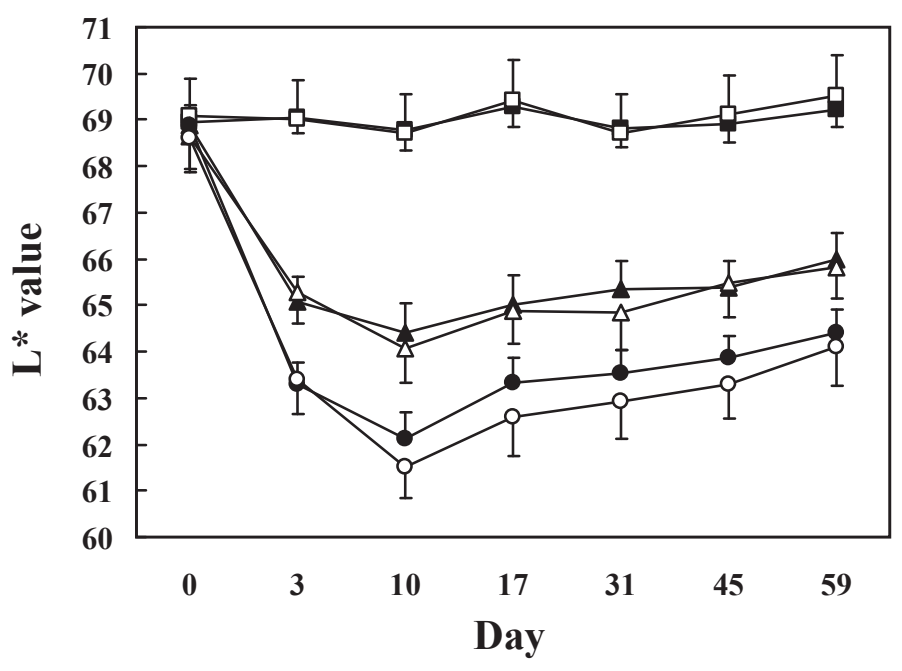

Fig. 2. Changes in skin lightness ( $\mathrm{L}^{*}$ value) at the sites of UV irradiation. Square, intact sites; triangle, irradiated 2,25 MED; circle, irradiated 3 MED.

Closed marker, test sample group; open marker, placebo group. Values are mean $\pm \mathrm{SE}$.

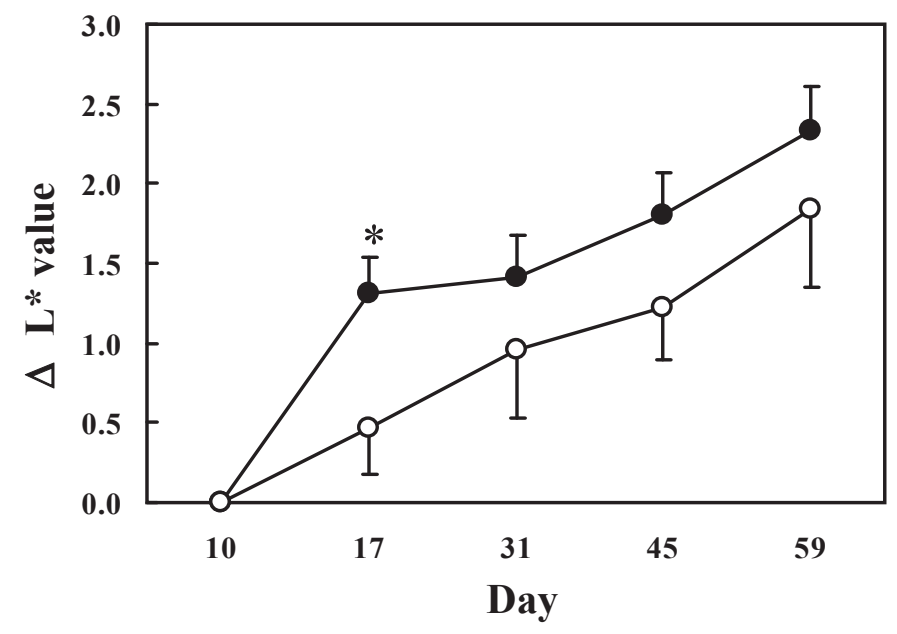

Fig. 3. Changes in skin lightness (L*value) from Day 10 (the lowest point) at the sites irradiated with 3 MED.

Closed marker, test sample group; open marker, placebo group. Values are mean \pm SE. Student's t-test $\quad *: p<0.05$.

\section{Discussion}

Numerous studies have been carried out to develop substances to prevent or reduce pigmentation in the skin, as it represents a serious cosmetic problem, especially for the elderly. Ascorbic acid, the most popular substance used in the prevention and treatment of pigment spots, is known to prevent melanogenesis by two mechanisms: 1) reducing dopaquinone, a precursor of melanin, and converting it into dopa, and 2) reducing oxidized melanin and converting it into reduced melanin. Substances such as arbutin, ${ }^{11)}$ kojic acid ${ }^{12)}$ and ellagic acid ${ }^{13)}$ prevent melanogenesis by inhibiting tyrosinase, which converts tyrosine into dopaquinone. Recently, it has been shown that ROS produced by UV light is highly relevant to skin pigmentation, and that therefore enhancing internal antioxidants is an effective method of preventing oxidative damage. Specifically, it was reported that supplementation of substances derived from foods, such as alphatocopherol, ascorbic acid and carotenoids, increases their serum concentrations and has a photo-protective effect on erythema development. ${ }^{14-18)}$

GSH is an intracellular antioxidant that plays an important role in protecting cells against oxidative stress. Moreover, GSH reinforces the reductive efficacy of ascorbic acid; it may switch the melanogenesis type from eumelanin to pheomelanin. ${ }^{5,6)} \mathrm{GSH}$ is also used for the treatment of skin pigmentation as a reducing agent with sulfhydryl. While intracellular GSH is expected to synthetically enhance the photo-protective effect of cells by a variety of mechanisms, it has not yet been confirmed that oral GSH intake increases intracellular GSH.

In the previous study, we found that olive leaf extract increases intracellular GSH in human fibroblasts, melanocytes and keratinocytes. The active compound in the extract was identified as hydroxytyrosol (3,4-dihydroxyphenyl ethanol), and it was confirmed that hydroxytyrosol also increases intracellular GSH in human fibroblasts, melanocytes and keratinocytes. We have shown in a previous study that hydroxytyrosol increases intracellular GSH by promoting an influx of cystine via the cystine/glutamate exchange transporter. Cystine is then converted to cystein by cystine reductase. Additionally, we showed that the oral administration of hydroxytyrosol decreased the number of dopapositive cells in the ear epidermis of mice exposed to UVB, and that the effect was more significant when hydroxytyrosol was administered in combination with cystine than alone. These results suggest that the oral ingestion of hydroxytyrosol has antimelanogenic effect and that cystine enhances this effect. Because GSH enhances the reductive efficacy of ascorbic acid, we hypothesized that the intake of olive leaf extract, L-cystine and ascorbic acid simultaneously would be effective in tanning prevention.

In our present study, we found that the MED significantly increased after ingestion of the test sample compared with that of non-ingested. It is suggested that the ingestion of the test sample prevented sunburn induced by UV irradiation. We believe that this prevention may be due to the antioxidative effect of hydroxytyrosol, oleuropein ${ }^{19)}$ and other polyphenols and flavonoids that directly scavenge ROS. The level of skin lightness after UV irradiation of the test sample group was slightly higher than that of the placebo group but the difference was not statistically significant. It is plausible that the ingestion of the test sample may prevent tanning by GSH, which switches the melanogenesis type from eumelanin to pheomelanin. The reason why the efficacy was not significant could be due to insufficient uptake by epidermal cells, since ingestion was initiated on the first day of UV irradiation. In most of the clinical studies on alphatocopherol and carotenoids, ingestion of the test substances started 
one week before UV irradiation, allowing serum concentrations to increase sufficiently. ${ }^{14-17)}$ Considering the mechanism by which the olive leaf extract intake increases intracellular GSH, it is expected that the efficacy may be higher if ingestion is initiated one week prior to irradiation. On the other hand, we also found that tanning improved faster in the test sample group than placebo group, which may be due to GSH reinforcing the reductive efficacy of ascorbic acid since the efficacy was exerted after intracellular GSH increased.

Olive leaf extract has been utilized as a complementary and alternative medicine, and no adverse events have been reported in conjunction with using it. In addition, no toxicity was observed in a single and repeated toxicity study and genotoxicity study of the extract used in this study. Given this, we believe olive leaf extract to be safe to use. The results in this study suggest that our supplement containing olive leaf extract, L-cystine and ascorbic acid is a safe and useful supplement for preventing sunburn and improving tanning. In this study, we studied a mixture of olive leaf extract, L-cystine and ascorbic acid based on our hypothesis of mechanism. Subsequent studies should investigate whether the ingestion of olive leaf extract alone exerts similar efficacy.

\section{References}

1) Mishima Y, Tanay A: The effect of alpha-methyldopa and ultraviolet irradiation on melanogenesis. Dermatologica 136;105-114:1968.

2) Quevedo WC, Szabo G, Virks J: Influence of age and UV on the populations of DOPA-positive melanocytes in human skin. J Invest Dermatol 52;279-284:1969.

3) Jimbow K, Kaidbey KH, Pathak MA, et al: Melanin pigmentation stimulated by UV-B, UV-A and psoralen. J Invest Dermatol 62;548:1974.

4) Meister A: Glutathione, ascorbate, and cellular protection. Cancer Res 54(Suppl.);1969-1975:1994.

5) Jara JR, Aroca P, Solano F, et al: The role of sulfhydryl compounds in mammalian melanogenesis: the effect of cysteine and glutathione upon tyrosinase and the intermediates of the pathway. Biochim Biophys Acta 967;296-303:1988.

6) Benedetto JP, Ortonne JP, Voulot C, et al: Role of thiol compounds in mammalian melanin pigmentation. II. Glutathione and related enzymatic activities. J Invest Dermatol 79;422-424:1982.

7) Connor MJ, Wheeler LA: Depletion of cutaneous glutathione by ultraviolet radiation. Photochemistry and Photobiology 46;239245:1987.

8) Rhie G, Shin MH, Seo JY, et al: Aging- and photoaging-dependent changes of enzymic and nonenzymic antioxidants in the epidermis and dermis of human skin in vivo. J Invest Dermatol 117;12121217:2001.

9) Yang CS, Chou ST, Liu L, et al: Effect of ageing on human plasma glutathione concentration as determined by high-performance liquid chromatography with fluorimetric detection. J Chromatogr B Biomed Appl 674;23-30:1995.

10) Khan Y, Panchal S, Vyas N, et al: Olea europaea: A PhytoPharmacological Review. Pharmacognosy Reviews 1:114-118:2007.
11) Tomita K, Fukuda M, Kawasaki K: Mechanism of arbutin inhibitory effect on melanogenesis and effect on the human skin with cosmetic use (in Janapese). Fragrance Journal 6;72-77:1990.

12) 12) Ohyama Y, Mishima Y: Melanogenesis-inhibitory effect of Kojic acid and its action mechanism (in Janapese). Fragrance Journal 6;53-58:1990.

13) Tachibana S, Tanaka Y: Inhibitory effect of ellagic acid on melanogenesis (in Janapese). Fragrance Journal 9;37-42:1997.

14) Mireles-Rocha H, Galindo I, Huerta M, et al: UVB photoprotection with antioxidants: Effects of oral therapy with d-alpha-tocopherol and ascorbic acid on the minimal erythema dose. Acta Derm Venereol 82;21-24:2002.

15) Stahl W, Heinrich U, Jungmann $\mathrm{H}$, et al: Carotenoids and carotenoids plus vitamin E protect against ultraviolet light-induced erythema in humans. Am J Clin Nutr 71;795-798:2000.

16) Heinrich U, Gartner C, Wiebusch M, et al: Supplementation with beta-carotene or a similar amount of mixed carotenoids protects humans from UV-induced erythema. J Nutr 133; 98-101:2003.

17) Lee J, Jiang S, Levine N, Watson RR. Carotenoid supplementation reduces erythema in human skin after stimulated solar radiation exposure. PSEBM 223;170-174:2000.

18) Ribaya-Mercado JD, Garmyn M, Gilchrest BA, Russell RM. Skin lycopene is destroyed preferentially over beta-carotene during ultraviolet irradiation in humans. J Nutr 125;1854-1859:1995.

19) Visioli F, Bellomo G, Galli C. Free radical-scavenging properties of olive oil polyphenols. Biochem Biophys Res Commun 247;6064:1998. 